\title{
Left recurrent laryngeal nerve palsy associated with primary pulmonary hypertension and recurrent pulmonary embolism
}

\author{
P T WILMSHURST, M M WEBB-PEPLOE, R J CORKER * \\ From the Department of Cardiology, St Thomas's Hospital, London, and Kent and Canterbury Hospital, Canterbury, \\ Kent
}

SUMmaRy Two patients are described with left recurrent laryngeal nerve palsy in association with pulmonary hypertension. One patient had recurrent pulmonary embolism and the other had primary pulmonary hypertension. A causal relation between pulmonary artery hypertension and the palsy is proposed.

Left recurrent laryngeal nerve palsy in association with pulmonary artery hypertension and dilatation as a result of chronic pulmonary embolism has been described once ${ }^{1}$ and with primary pulmonary hypertension only rarely. ${ }^{2-6}$ We present an additional two patients; one with each of these causes of pulmonary hypertension.

\section{Case 1}

A 25 year old man presented in October 1980 with a two month history of dyspnoea, cough, haemoptysis, chest pain, lethargy, and hoarseness. He was a nonsmoker. He was in sinus rhythm, with blood pressure $90 / 60 \mathrm{mmHg}$, venous pressure raised $8 \mathrm{~cm}$, prominent right ventricular and pulmonary artery impulses, loud $P_{2}$, and the murmur of tricuspid regurgitation. Chest $x$-ray film showed enlarged main pulmonary arteries with reduced vascular markings in both lower zones and right upper zone. The electrocardiogram showed right ventricular hypertrophy and right bundle-branch block, and echocardiography showed a normal left heart but dilated right ventricle, septal paradox, and mid-systolic notching of pulmonary valve. Hilar tomography and computerised axial tomography (CAT) scan of the chest showed only enlarged pulmonary arteries. Bronchoscopy was normal apart from paralysis of the left vocal cord. Routine haematology, biochemistry, autoantibodies, intravenous urography, barium swallow and meal,

^Present address: Department of Radiology, King's College Hospital, London. and CAT scan of abdomen and pancreas were unhelpful.

Cardiac catheterisation was performed and haemodynamic data are shown in the Table. Left ventricular cineangiography and selective coronary angiography were normal. Selective bronchial arteriograms showed drainage into the pulmonary venous sytem. Pulmonary angiograms confirmed dilated main pulmonary arteries with reduced blood flow to both lower zones and right upper zone, with thrombus seen (Fig.). Phlebography of arms and legs showed no evidence of thrombus.

He was treated with diuretics and warfarin without clinical improvement. In April 1981 he developed acute right sided chest pain, dyspnoea, cyanosis, tachycardia, and died. Necropsy confirmed a recent embolic right pulmonary infarct, with right ventricular hypertrophy and failure. The left recurrent laryngeal nerve appeared normal, as did the rest of the necropsy.

\section{Case 2}

A 37 year old Asian man was admitted in May 1981 with a 12 month history of progressive abdominal distension, dyspnoea, cough, fatigue, and weight loss. His voice had been hoarse for eight months. He had not had chest pain, haemoptysis, or ankle swelling. He had a long history of alcohol abuse, but had never smoked. He was in sinus rhythm, with blood pressure $100 / 80 \mathrm{mmHg}$, venous pressure raised $10 \mathrm{~cm}$, prominent right ventricular and pulmonary artery impulses, loud $P_{2}$, a pulmonary flow murmur, and the signs of tricuspid regurgitation, with gross ascites. Chest $x$-ray film showed enlargement of the heart and main pul- 
Table Haemodynamic data

\begin{tabular}{lll}
\hline & \multicolumn{2}{l}{ Pressures (mmHg) } \\
\cline { 2 - 3 } & Case l & Case 2 \\
\hline Right atrium & 1 to $7(4)$ & 6 to $17(13)$ \\
Right ventricle & $82 / 1$ to 12 & $80 / 8$ to 10 \\
Pulmonary artery & $82 / 36(53)$ & $80 / 43(57)$ \\
Left atrium & -2 to $3(0)$ & -1 to $13(5)$ \\
Left ventricle & $94 /-2$ to 2 & $85 /-4$ to 5 \\
Aorta & $94 / 70(82)$ & $85 / 66(74)$ \\
Indocyanine green dye curves & & \\
Cardiac output (1/min) & $4 \cdot 4$ & $2 \cdot 2$ \\
Intracardiac shunting & No & No \\
Bronchopulmonary shunting & $26 \%$ of & \\
& \multicolumn{2}{c}{ pulmonary flow No } \\
\hline
\end{tabular}

Means are given in parentheses.

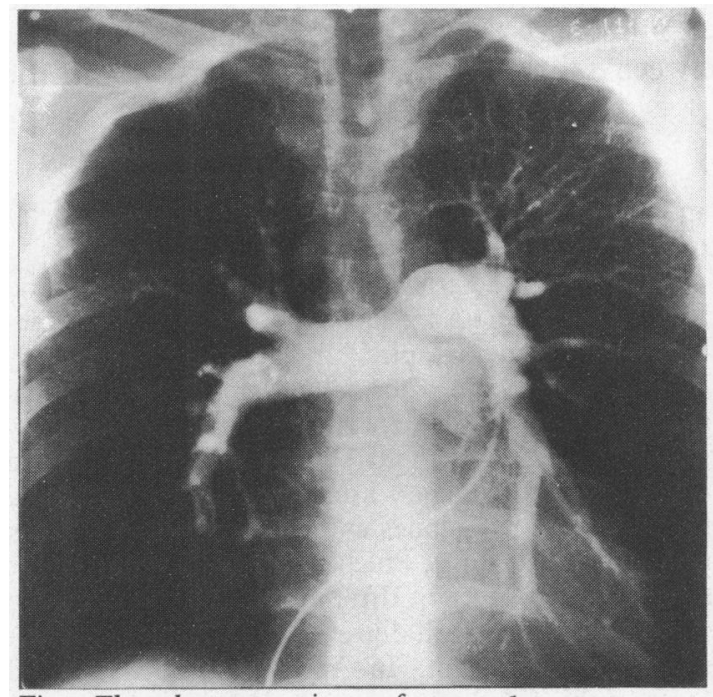

Fig. The pulmonary angiogram from case 1 .

monary arteries. The vascular markings were reduced in the periphery of both lung fields. The electrocardiogram showed first degree heart block, right atrial and right ventricular hypertrophy, right bundlebranch block, and $Q R S$ axis $+165^{\circ}$. The left heart was normal on echocardiography, but the right ventricle was dilated, with septal paradox and a pericardial effusion. Bronchoscopy was normal apart from paralysis of the left vocal cord. Routine haematology, autoantibodies, and isotope liver scan were unremarkable. Abdominal ultrasound confirmed gross ascites and dilated hepatic veins, but no space occupying lesion. Liver function tests were slightly abnormal and liver biopsy (transjugular) showed sinusoidal dilatation and centrilobular fibrosis.

Cardiac catheterisation was performed and haemodynamic data are set out in the Table. Pulmonary angiography showed dilated central pulmonary arteries, with peripheral pruning.

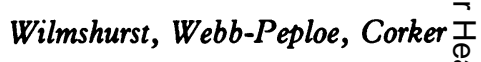

Despite treatment with diuretics, anticoagulants, and vasodilators he deteriorated and died in October 1981. Necropsy confirmed the diagnosis of primary $\stackrel{\vec{S}}{\rightarrow}$ pulmonary hypertension with right heart failure. Theo left recurrent laryngeal nerve was grossly normal, as was the rest of the necropsy.

\section{Discussion}

Ortner $^{7}$ in 1897 was first to report the association. between mitral valve disease and paralysis of the left $\vec{\omega}$ recurrent laryngeal nerve. It is now realised that this $\stackrel{ }{\circ}$ association occurs more frequently than could be expected by chance. ${ }^{8}$ The aetiology of the palsy wase originally attributed to compression of the nerve by the dilated left atrium of these patients. ${ }^{7}$ More recently others have suggested that compression of $\rightarrow$ the nerve between the dilated, hypertensive pulmo- nary artery and the aorta is the cause.$^{8}$ In all caseswhere measured, the pulmonary arterial pressure was ${ }_{\mathbb{\Phi}}^{\text {T }}$ raised, and the palsy may be relieved by mitral valve을 surgery if the pulmonary arterial pressure is reduced. ${ }^{8}$ Left recurrent laryngeal nerve palsy has occasionally $₹$ been reported in patients with primary pulmonary $\overrightarrow{0}$ hypertension $^{2-6}$ and in one patient with recurrent $\omega$ pulmonary emboli. ${ }^{1}$ In only four of the patients ${ }^{13-5}$. was pulmonary arterial pressure measured, and in only three patients 235 was there histological confirmation of the underlying pulmonary pathology at necropsy.

Primary pulmonary hypertension is uncommon. $\stackrel{2}{\Rightarrow}$ Acute pulmonary embolism is common, but it is rare윽 for recurrent pulmonary embolisation to give rise to chronic pulmonary hypertension. The presentation, at our hospital, during a 12 month period, of two patients with pulmonary hypertension as a result of $\widetilde{\mathbb{C}}$ these two conditions associated with left recurrent? laryngeal nerve palsy is, we feel, more than coinciden-음 tal. During a similar interval of time two patients ${ }^{2} 3$. presented at Guy's Hospital with primary pulmonaryo hypertension and left recurrent laryngeal nerve palsy. Patients with primary pulmonary hypertension and? chronic pulmonary embolisation, unlike those with $\rightarrow$ mitral valve disease, do not have enlarged left atria. Compression of the recurrent laryngeal nerve by this structure cannot be implicated in the aetiology of the palsy. In our patients compression of the nerve between two high pressure vessels, aorta and pulmonaryw artery, seems more probable, and the time course of the onset of the hoarseness of voice supports such ao causal relation. These case reports also support theo view that the nerve palsy in Ortner's syndrome is ${ }^{\text {? }}$ caused by compression of the nerve by the pulmonary artery and not the left atrium.

Recognition that an association may occur between recurrent laryngeal nerve palsy and pulmonary hyper $\frac{\mathbb{}}{2}$ 
tension, for reasons other than those of mitral disease, may save such patients from unnecessary investigations.

\section{References}

1 Albertini RE. Vocal cord paralysis associated with pulmonary emboli. Chest 1972; 62: 508-10.

2 Brinton WD. Primary pulmonary hypertension. $\mathrm{Br}$ Heart f 1950; 12: 305-11.

3 Soothill JF. A case of primary pulmonary hypertension with paralysed left vocal cord. Guys Hosp Rep 1951; 100: 232-7.

4 Böhme H, Böhme G. Das Ortner-Syndrom. Munch Med Wochenschr 1962; 104: 681-6.
5 Rosenberg SA. A study of the etiological basis of primary pulmonary hypertension. Am Heart $\mathcal{f}$ 1964; 68: 484-9.

6 Kagal AE, Shenoy PN, Nair KG. Ortner's syndrome associated with primary pulmonary hypertension. $\mathcal{f}$ Postgrad Med 1975; 21: 91-5.

7 Ortner N. Recurrenslähmung bei Mitralstenose. Wien Klin Wochenschr 1897; 10: 753-5.

8 Camishion RC, Gibbon JH Jr, Pierucci L Jr, Lida J. Paralysis of the left recurrent laryngeal nerve secondary to mitral valvular disease. Ann Surg 1966; 163: 818-28.

Requests for reprints to Dr P T Wilmshurst, Department of Cardiology, St Thomas's Hospital, London SE1 7EH. 